\title{
Heart block in patients with sleep apnoea
}

\author{
H F Becker, U Koehler, A Stammnitz, J H Peter
}

\section{Prevalence of bradycardic arrhythmias in sleep apnoea}

In the early days of sleep research it was thought that heart block (second and third degree atrioventricular block), sinus arrest, or sinoatrial block were frequent findings in patients with sleep apnoea. Tilkian and co-workers ${ }^{1}$ reported that more than $50 \%$ of patients with sleep apnoea develop episodes of heart block during sleep. In 400 patients studied by Guilleminault et $a l^{2}$ bradycardic arrhythmias were reported in almost $18 \%$ of the patients and later studies found heart block in 9-13\% of patients with sleep apnoea. ${ }^{34}$ Due to ventricular asystoles of up to 13 seconds in duration it was hypothesised that heart block might lead to an increased mortality risk in these patients. ${ }^{2}$ A recent publication challenged previous results in that no increased prevalence of bradycardic arrhythmias was seen in patients with sleep apnoea compared with those without sleep apnoea. ${ }^{5}$

To examine these seemingly discrepant results further we performed Holter monitoring in 239 consecutive patients diagnosed as having sleep apnoea (apnoea/hypopnoea index $>10 / \mathrm{h}$ ) using a validated ambulatory recording device based on the measurement of heart rate, $\mathrm{SaO}_{2}$, snoring sound, and body position. Episodes of second and third degree atrioventricular (AV) block and/or sinus arrest of more than two seconds in duration occurred in 17 of the 239 patients. ${ }^{6}$ There was no significant difference in age between patients with and without heart block (mean (SD) 50.7 (12.8) versus 52.1 (9.8) years), but those with heart block were significantly more obese (38.7 (7.3) versus 30.7 (4.6)).

Twelve of the 17 patients $(70.6 \%)$ had arterial hypertension, seven $(41.1 \%)$ suffered from heart failure (NYHA II-III), and four $(23.5 \%)$ had pulmonary hypertension. None of the patients reported a myocardial infarction in their medical history, and in only one $(5.9 \%)$ exercise testing showed signs of coronary heart disease. Ten of the 17 patients received no medication at all. Treatment with digoxin in two patients and with a $\beta$ blocker in one patient was discontinued one week prior to the study. Diuretic therapy in four patients and ACE inhibitors in two patients were continued unchanged.

Standard polysomnography and a repeat 24 hour Holter monitoring were then performed. Severe sleep apnoea (mean (SD) AHI 90 $(36) / h$, range 59.6-158) was found in the 17 patients and in all of them episodes of heart block during sleep could again be demonstrated. In two of these patients clusters of sinus arrest and second degree AV block could be demonstrated during the daytime as well.

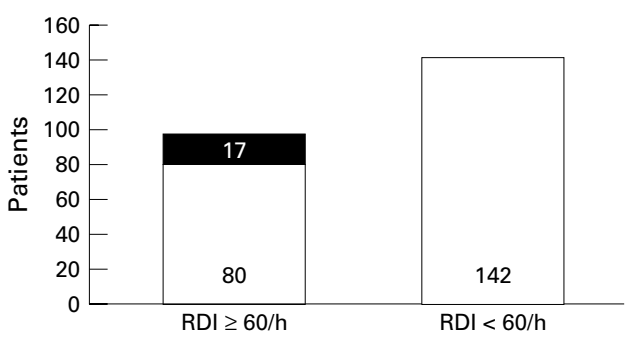

Figure 1 Number of patients with bradycardic arrhythmias in patients with $\geqslant 60$ or $<60$ apnoeas and hypopnoeas per hour of sleep (RDI).

Both patients reported that they were sleeping at the times when heart block had occurred.

A total of 10368 apnoeas and hypopnoeas were recorded, $81.8 \%$ of which were obstructive and mixed apnoeas, $17 \%$ were obstructive hypopnoeas, and only $1.2 \%$ were central apnoeas. Bradycardic arrhythmias exclusively occurred during apnoeas and hypopnoeas and were not found during hyperventilation.

The occurrence of bradycardic arrhythmias was clearly linked to apnoea severity: none of the patients with an AHI below 60/h developed heart block, whereas 17 of the 97 with an AHI of at least $60 / \mathrm{h}$ did (fig 1). ${ }^{6}$ Although the AHI threshold of $60 / \mathrm{h}$ cannot be taken as an absolute value, bradycardic arrhythmias seem to occur exclusively in severe sleep apnoea; $20 \%$ of the patients with an AHI of at least $60 / \mathrm{h}$ developed heart block during sleep. Thus, the discrepant results concerning the prevalence of heart block in sleep apnoea are probably caused by the differences in severity of the disease in these studies.

\section{Mechanisms}

Changes in heart rhythm are a typical finding in sleep apnoea and are present in most patients. The characteristic pattern is the so called cyclical variation of heart rate with bradycardia during the apnoea followed by tachycardia during the post-apnoeic hyperventilation. ${ }^{27}$ The cycle length of respiratory sinus arrhythmia is only four or five heart beats, whereas that of the apnoea associated bradycardic and tachycardic phases is longer as it is linked to the length of the apnoeas, being $10-60$ seconds in duration.

It is a well known clinical finding that hypoxaemic patients - for example, those with pulmonary oedema, pneumonia, or asthma-also present with tachycardia. However, hypoxia leads to bradycardia in the absence of lung inflation. ${ }^{8}$ This is the case in both central and obstructive apnoeas. One study in patients with obstructive sleep apnoea (OSA) reported a decrease in heart rate during apnoeas in REM sleep but an increase in heart rate during apnoeas in non-REM sleep. ${ }^{9}$ When hypoxia 
during apnoeas is prevented by oxygen breathing, bradycardia does not occur or is markedly reduced. ${ }^{8}$ The physiological significance of bradycardia during apnoea induced hypoxia might be a reduction in oxygen consumption by the heart, as is regularly demonstrated before or during birth in humans if the fetus is hypoxic and also in diving mammals.

In patients with sleep apnoea Tilkian and co-workers ${ }^{1}$ demonstrated that cyclical variation of heart rate and even episodes of heart block could be prevented by administration of atropine without affecting the apnoeas. Thus, hypoxia in the absence of lung inflation seems to cause vagal stimulation leading to bradycardia, which can be prevented by vagal blockade with atropine.

Although these mechanisms have been generally accepted, the question remains as to why some patients develop heart block and others do not. Only 17 out of 97 patients with severe sleep apnoea (AHI at least 60/h) developed bradycardic arrhythmias. ${ }^{6}$

Guilleminault reported that bradycardic arrhythmias exclusively occurred during apnoeas with an $\mathrm{SaO}_{2}$ below $72 \% .^{2}$ In the detailed analysis of our data $^{6}$ we could not confirm this finding. Episodes of heart block were found in $15.2 \%$ (1575) of the 10368 apnoeas and hypopnoeas seen in our 17 patients. The percentage of apnoeas/hypopnoeas in which heart block occurred increased with the degree of desaturation and was $6.6 \%, 8.0 \%, 18.7 \%$, and $19.6 \%$ if

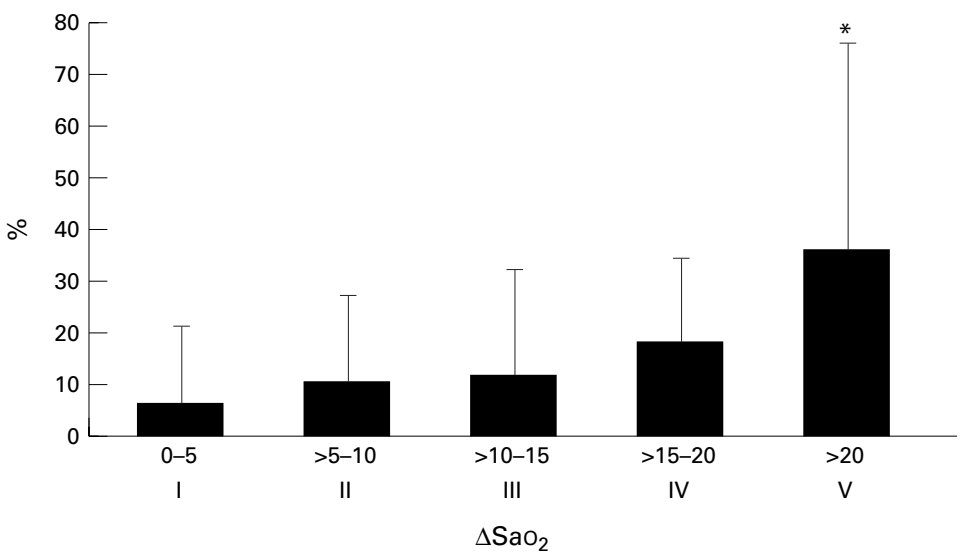

Figure 2 Effect of desaturation on the occurrence of heart block, expressed as mean (SD) percentage of apnoeas and hypopnoeas with heart block in different desaturation classes.

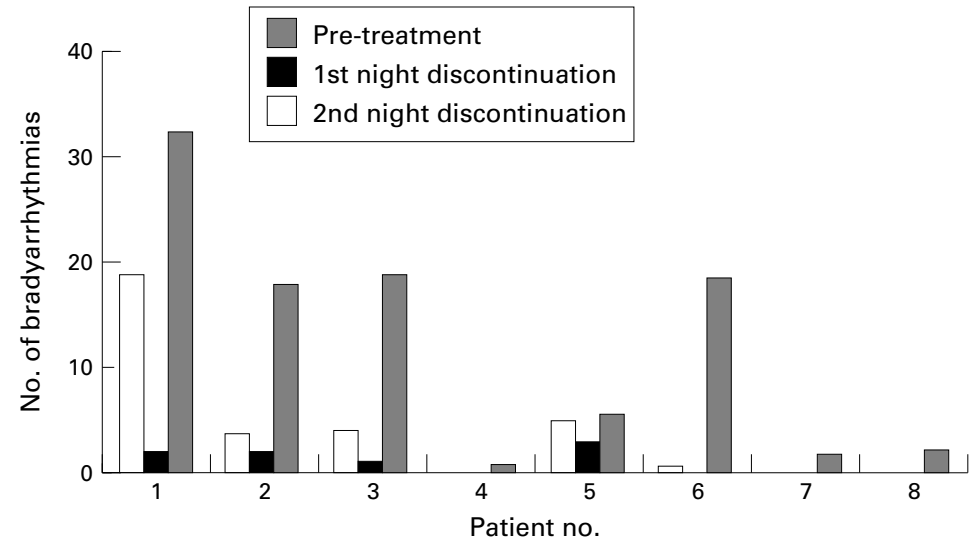

Figure 3 Recurrence of bradycardic arrhythmias after discontinuation of nCPAP therapy. the $\mathrm{SaO}_{2}$ nadir was $\geqslant 90 \%$, $\geqslant 80$ to $<90 \%$, $\geqslant 70$ to $<80 \%$, and $<70 \%$, respectively. Thus, there is a clear tendency that heart block is more likely to occur with greater desaturation. However, we could not find a threshold below which heart block exclusively occurred; $46 \%$ of the episodes of heart block occurred during apnoeas with a minimal $\mathrm{SaO}_{2}$ of $72 \%$ or more.

Due to the large interindividual variability of the number of bradyarrhythmias, any analysis of the influence of desaturation on the occurrence of heart block has to take this variability into account. We therefore defined five desaturation classes and calculated the percentage of apnoeas with bradycardic arrhythmias for each patient in each desaturation class (fig 2). On average, bradycardic arrhythmias occurred in a mean (SD) of 6.2 (15)\% of apnoeas with a desaturation of $5 \%$ or less. With more pronounced desaturation the percentage of apnoeas in which heart block occurred increased to a maximum of 35.6 (39) \% in apnoeas with a desaturation of more than $20 \%$. However, due to the large variability, only the difference between groups I and V was statistically significant $(\mathrm{p}<0.05)$.

There are few data on the influence of sleep stages on heart block. There was a substantial increase in the number of bradycardic arrhythmias during REM sleep in our patients. ${ }^{10}$ Before treatment $89.2 \%$ of the episodes of heart block occurred during REM sleep, but this sleep stage accounted for only $20 \%$ of the total sleep time. However, as apnoeas are generally longer in REM than in non-REM sleep, desaturation is more pronounced. Therefore, in 17 patients $^{6}$ we performed covariant analysis adjusting for desaturation and apnoea duration. This revealed that REM sleep is an independent factor contributing to heart block in these patients ( $\mathrm{p}<0.05)$.

Eight patients in whom episodes of heart block were diagnosed before long term nasal continuous positive airway pressure (nCPAP) therapy was initiated were re-studied with polysomnography and Holter monitoring after an average of 14 months on nCPAP. There were no bradyarrhythmias while patients were on nCPAP. After discontinuation of the treatment for two nights the AHI and the mean duration of the apnoeas were virtually identical to the pretreatment values. In five patients the mean (SE) number of episodes of heart block present after two nights was 4.3 (2.3) without nCPAP and 13.5 (4.5) before treatment (fig 3 ).

In patients with coronary heart disease and sleep apnoea bradycardic arrhythmias may occur during sleep. ${ }^{11}$ However, coronary heart disease is a rare finding in patients with obstructive sleep apnoea and sleep related heart block ${ }^{12}$ and only one of our 17 patients had signs of it. Thus, coronary heart disease does not seem to play an important part in the pathogenesis of bradycardic arrhythmias in sleep apnoea.

Although REM sleep and excessive vagal activation due to hypoxia and apnoea are important mechanisms leading to bradycardic arrhythmias in these patients, the question still remains unanswered as to why only approximately $20 \%$ 
of the patients with severe OSA develop heart block. An abnormal parasympathetic responsiveness might also be an additional contributing factor to heart block.

\section{Electrophysiological studies}

To our knowledge, only two articles have been published on electrophysiological studies in patients with OSA and heart block. In the 1970s Tilkian et al ${ }^{1}$ reported on electrophysiological studies in five patients with OSA, two of whom had episodes of heart block of more than three seconds duration. In these patients no abnormalities of the conduction system were detected during wakefulness. Recently published data in 15 consecutive patients with OSA and ventricular asystole of at least five seconds in duration also show that structural changes of the conduction system are not present in these patients. ${ }^{13}$ However, the study showed mild abnormalities in more than $50 \%$ of the patients at rest: prolonged sinoatrial conduction times in two patients, atrioventricular nodal function abnormalities in eight patients (53\%), and slightly prolonged His ventricular conduction time in nine patients. ${ }^{13}$ The fact that these mild abnormalities were completely reversible with atropine administration in all but two patients ${ }^{13}$ further supports the concept of an increased vagal tone as the most important pathological mechanism leading to heart block in these patients.

\section{Effect of nCPAP therapy}

As heart block is clearly caused by sleep disordered breathing in this patient group, treatment of sleep apnoea should be the primary therapeutic strategy. Tracheotomy has been shown to be an effective treatment for sleep apnoea and heart block in a small group of patients, but due to its side effects is now only used rarely. Nasal CPAP has become the standard treatment for patients with sleep apnoea. With this treatment the number of bradycardic arrhythmias was reduced significantly from 1575 to 165 episodes per night in 17 patients. ${ }^{6}$ In 12 of the patients heart block was completely prevented and markedly reduced in another three with one or two nights of effective nCPAP therapy. With treatment, $81 \%$ of the episodes of heart block occurred during hypopnoeas due to mouth leaks with ineffective treatment pressure, during central apnoeas ( $1 \%)$, but also during normal breathing in REM sleep (18\%). After four weeks of nCPAP two patients still presented repetitive episodes of heart block despite effective nCPAP therapy. A second study in another 15 patients confirmed these data, demonstrating reversal of heart block with nCPAP in $87 \%$ of the patients. ${ }^{13}$

\section{Pacemaker therapy}

In contrast to tracheotomy, nCPAP treatment may be discontinued or interrupted. Both the breathing disorder ${ }^{14-16}$ and episodes of heart block will re-occur after the cessation of treatment in most patients. The use of pacemaker therapy in addition to nCPAP might therefore be considered. Our clinical practice is to recommend using a pacemaker if relevant bradycardic arrhythmias persist despite nCPAP, or if compliance with nCPAP seems unsatisfactory.

\section{Sleep apnoea in asymptomatic patients} with heart block

Heart block may be present in seemingly healthy and athletic young adults. Second degree AV block type Wenckebach and episodes of sinus arrest of approximately two seconds in duration have been reported. ${ }^{17}{ }^{18}$ As polysomnography was not performed in these subjects without obvious heart disease, OSA might have caused heart block at least in some of them. In a recently published study polysomnography was performed in eight patients with asymptomatic bradyarrhythmias (no syncope or lightheadedness) who had been referred to an electrophysiology practice for pacemaker therapy. ${ }^{19}$ Seven of the eight patients were diagnosed as having OSA and the episodes of heart block were prevented with treatment in all seven patients. This study confirms that patients with bradycardic arrhythmias occurring predominantly during sleep should be treated with further measures to exclude sleep apnoea as the underlying disease. $^{2420}$

\section{Conclusions}

In patients presenting with asymptomatic intermittent bradycardic arrhythmias, sleep apnoea should be considered as a possible cause. Cyclical variation of the heart rate is present in most patients. Episodes of heart block can be expected in approximately $20 \%$ of patients with severe sleep apnoea (AHI $>60 / \mathrm{h}$ ) and approximately $7.5 \%$ of an unselected group of patients with OSA. There is no threshold below which bradyarrhythmia exclusively occurs, although the risk increases with the amount of desaturation. REM sleep is an independent factor leading to heart block, irrespective of apnoea duration and desaturation. As the duration of the asystole seen in these patients is often considerable, heart block might constitute an important risk factor for sudden death in patients with sleep apnoea. Effective treatment with nCPAP leads to a complete prevention of heart block in $80-90 \%$ of these patients.

\footnotetext{
1 Tilkian AG, Guilleminault C, Schroeder JS, et al. Sleepinduced apnea syndrome. Prevalence of cardiac arrhythmias and their reversal after tracheostomy. Am $\mathcal{F} \mathrm{Med}$ 1977;63:348-58.

2 Guilleminault C, Connolly SJ, Winkle RA. Cardiac arrhythmia and conduction disturbances during sleep in 400 patients with sleep apnea syndrome. Am f Cardiol 1983;52: $490-4$

3 Bolm-Audorff U, Köhler U, Becker E, et al. Nächtliche Herzrhythmusstörungen bei Schlafapnoe-Syndrom. Dtsch Med Wschr 1984;109:853-6.

4 Miller WP. Cardiac arrhythmias and conduction disturbances in the sleep apnea syndrome. Prevalence and significance. Am F Med 1982;73:317-21.

5 Flemons WW, Remmers JE, Gillis AM. Sleep apnea and cardiac arrhythmias. Is there a relationship? Am Rev Respir Dis 1993;148:618-21.

6 Becker H, Brandenburg U, Peter JH, et al. Reversal of sinus arrest and atrioventricular conduction block in patients with sleep apnea during nasal continuous positive airway with sleep apnea during nasal continuous positive a

7 Tilkian A, Guilleminault C, Schroeder JS, et al. Hemodynamics in sleep induced apnea: studies during wakefulness and sleep. Ann Intern Med 1976;85:714-9.
} 
8 Zwillich C, Devlin T, White D, et al. Bradycardia during sleep apnea Characteristics and mechanism. 7 Clin Invest sleep apnea. Charact

9 Stoohs R, Guilleminault C. Cardiovascular changes associated with obstructive sleep apnea syndrome. 7 Appl Physiol 1992;72:583-9.

10 Becker H, Brandenburg U, Conradt R, et al. Einflub der nCPAP-Therapie auf bradykarde Herzrhythmusstörungen bei Schlafapnoe (SA). Pneumologie 1993;47:706-10.

11 Köhler U, Dubler H, Glaremin T, et al. Nocturnal myocardial ischemia and cardiac arrhythmia in patients with sleep apnea with and without coronary heart disease. Klin Wschr 1991;69:474-82.

12 Köhler U, Wetzig T, Peter JH, et al. Morbiditat und Letalitat bei Schlafapnoe und nachtlichen Bradyarrhythmien. Dtsch Med Wschr 1994;119:1187-93.

13 Grimm W, Hoffmann J, Menz V, et al. Electrophysiologic evaluation of sinus node function and atrioventricular conduction in patients with prolonged ventricular asystole during obstructive sleep apnea. Am f Cardiol 1996;77: during $1310-4$.

14 Kribbs NB, Pack AI, Kline LR, et al. Effects of one night without nasal CPAP treatment on sleep and sleepiness in patients with obstructive sleep apnea. Am Rev Respir Dis 993;147:1162-8.

15 Rolfe I, Olson -8. . does not ameliorate obstructive sleep apnea. Aust NZ 7 Med 1991;21:235-8.

16 Brandenburg U, Weiner M, Becker $\mathrm{H}$, et al. Hämodynamik und Schlafstruktur im Auslabversuch nach nCPAPBeatmungstherpie bei obstruktivem Schlafapnoesyndrom. Pneumologie 1993;47(Suppl 1):181-3.

17 Bjerregaard P. Mean 24 hour heart rate, minimal heart rate and pauses in healthy subjects $40-79$ years of age. Eur Heart $\mathcal{F} 1983 ; 4: 44-51$

18 Brodsky M, Delon WU, Denes P, et al. Arrhythmias documented by 24 hours continuous electrocardiographic monitoring in 50 male medical students without apparent heart disease. Am 7 Cardiol 1977;39:390-5.

19 Morgan BJ, Crabtree DC, Puleo DS, et al. Neurocirculatory consequences of abrupt change in sleep state in humans. $\mathcal{f}$ consequences of abrupt change

20 Köhler U, Funck R, Fus E, et al. Bradykarde Herzrhythmusstörungen bei Schlafbezogenen Atmungsstörungen: Therapie. Dtsch Med Wschr 1996;129:1369-73. 